\title{
Red blood cell microrheological effects of some antitumor chemotherapy drugs: In vitro study
}

\author{
A.V. Muravyov ${ }^{\mathrm{a}, *}$, I.A. Tikhomirova ${ }^{\mathrm{a}}$, N.V. Kislov ${ }^{\mathrm{b}}$ and A.S. Petrochenko \\ ${ }^{a}$ State Pedagogical University, Yaroslavl, Russia \\ ${ }^{\mathrm{b}}$ Regional Oncological Hospital, Yaroslavl, Russia
}

\begin{abstract}
In consequence of limitation of capillary dilatation reserve the role of erythrocyte membrane elasticity and their microrheological properties is very important for the tissue perfusion. The drugs that enter blood flow could be as signaling molecules and their action can lead to alteration of red blood cell aggregation and whole cell deformability. Therefore the subject of this study was to estimate the effect of some antitumor chemotherapy drugs on red blood cell aggregation (RBCA) and deformability (RBCD). It was found that an exposure of red blood cells (RBCs) to cisplatin and epoetin alfa led to significant positive changes in the RBC microrheological properties. It was shown that cisplatin could activate tyrosine protein kinase (TPK). Preincubation of RBCs with the inhibitor of EGF-R and Src kinase lavendustin A was accompanied by a significant decrease of the cisplatin effect. It was found RBC aggregation rise after cell incubation with 5-fluorouracil. This effect was removed with $\mathrm{Ca} 2+$ chelation with EGTA and also with pentoxifylline. The drug for the targeted antitumor therapy sunitinib raised RBCA markedly. Whereas RBC deformability index was altered only slightly. The combination of two chemicals: sunitinib and cisplatin led to an elimination of proaggregative effect of sunitinib.

Examination of the effects of some antitumor drugs on red blood cell microrheological properties has enabled us to suggest that the observed changes in red cell aggregation and deformability may be partly related to the effects of tyrosine protein kinase activation. It is accompanied by the alteration of red blood cell microrheology and the blood transport efficacy.
\end{abstract}

Keywords: Red blood cells, deformability, aggregation, chemotherapy, cisplatin, sunitinib, 5-fluorouracil, epoetin alfa

\section{Introduction}

In chemotherapy and immunotherapy, blood flow plays an important role in the delivery of appropriate pharmacological agents. In consequence of limitation of capillary dilatation reserve the role of erythrocyte membrane elasticity and their microrheological properties is very important for the tissue perfusion [8]. The drugs that enter blood flow under systemic chemotherapy could be as signaling molecules and their action can lead to an alteration of red blood cell microrheological properties and blood transport efficacy $[16,17,19]$. If the microrheological response on drug is positive then it is possibility to improve the drug transport and delivery into the tumor microvascular network. Cisplatin is one of the most potent antitumor agents known, displaying clinical activity against a wide variety of solid tumors [22, 29]. It can act as the tyrosine protein kinase (TPK) activator [9, 23]. The last decade has seen the introduction of several new classes of targeted anticancer therapies for routine clinical use $[10,28]$. These small molecules typically block pathways that are continuously activated in cancer cells. The tyrosine kinase inhibitors are the most common and work by inhibiting kinases that phosphorylate key proteins to activate signal transduction pathways [25]. It is known that red cell membrane

*Corresponding author: A.V. Muravyov, State Pedagogical University, Yaroslavl, Russia. Tel.: +0074852728633; Fax: +0074852305596; E-mail: alexei.47@mail.ru. 
contains tyrosine protein kinase $[9,13,14,18]$. Therefore we can suppose that drugs with TPK activity can act directly on the red blood cell microrheological properties. Even though 5-fluorouracil (FU) is one of the oldest anticancer drugs, its use in cancer chemotherapy continues to increase. Epoetin, administered at standard dosages has been shown to significantly increase hemoglobin levels, decrease transfusion requirements, and improve quality-of-life parameters in patients undergoing chemotherapy [7]. It is known epoetin activates many distinct signal transduction cascades, including a stimulation of tyrosine kinase - JAK-kinases [2, 31]. These chemicals induce protein tyrosine phosphorylation of a number of cellular proteins, which suggests involvement of protein tyrosine kinases in the activation process of red cell membrane $[5,16,27]$. Therefore the subject of this study was to estimate the effect of above-mentioned chemotherapy drugs on red blood cell deformability, aggregation and their suspension fluidity.

\section{Materials and methods}

\subsection{Preparation of blood samples}

Venous blood samples $(15 \mathrm{ml})$ were drawn via sterile venipuncture using heparin $(5 \mathrm{IU} / \mathrm{ml})$ as anticoagulant. The study was approved by the local ethic committee at the Yaroslavl State Pedagogical University, and an informed consent of all the subjects were obtained according to the recommendations of the Declaration of Helsinki (The International Response to Helsinki VI, The WMA's Declaration of Helsinki on Ethical Principles for Medical Research Involving Human Subjects, as adopted by the 52nd WMA General Assembly, Edinburgh, October, 2000).

Red blood cells (RBCs) were separated by centrifugation at $1,400 \mathrm{~g}$ for $20 \mathrm{~min}$ and washed 3 times with $10 \mathrm{mM}$ phosphate buffered saline $(\mathrm{pH}=7.4)$. The washed $\mathrm{RBCs}$ were then resuspended in the final medium (Dextran-200 and Ringer's solution in ratio 3:7) at a hematocrit of $40.0 \pm 0.3 \%$ for incubation with drugs and without ones (control). After an incubation period the supernatant was removed by centrifugation and cell suspensions were ready for red blood cell aggregation and deformability measurements.

\subsection{Protocols for in vitro aggregation studies}

It was organized three research sessions. In the first one RBC suspension was divided into three aliquots and exposed to: 1) Cisplatin (cis-dichlorodiammine platinum), a potent chemoimmunotherapeutic drug $(3.0 \mu \mathrm{M})$. It induces protein tyrosine phosphorylation of a number of cellular proteins suggesting the involvement of protein tyrosine kinase(s) in the activation process of the cells [23]; 2) 5-fluorouracil $(5-\mathrm{FU}, 5.0 \mu \mathrm{M})$ is one of the oldest anticancer drugs, its use in cancer chemotherapy continues to increase; 3 ) The red blood cells, incubated in Ringer's solution without any drugs, were used as a control.

In the second research session RBC suspension was divided into two aliquots and exposed to: 1) sunitinib (Sunitinib malate, $1.0 \mu \mathrm{M}$ ) - targeted antitumor drug, inhibitor of cellular signaling by targeting multiple receptor tyrosine kinases (RTKs); 2) epoetin alfa - recombinant human erythropoietin $(10 \mathrm{U} / \mathrm{ml})$.

In the third research session RBC suspension was divided into several aliquots and exposed to: 1) cisplatin alone and in combined with of lavendastin A (a selective tyrosine kinase inhibitor, $3.0 \mu \mathrm{M}$ ); 2 ) the combination of sunitinib and cisplatin; 3) the combination of 5-FU and EGTA (ethylene glycol tetraacetic acid) is a $\mathrm{Ca} 2+$ chelating agent $(1.0 \mathrm{mM}) ; 4)$ the combination of 5-FU and pentoxifylline $(1.0 \mu \mathrm{M})$. 
The cell shape was controlled with the light microscopy. The cell incubation was performed at $37^{\circ} \mathrm{C}$ for $30 \mathrm{~min}$. The red blood cells, also incubated in the final medium (Dextran-200 and Ringer's solution in ratio 3:7) without any drugs, were used as a control. Stock solutions of drugs were prepared in DMSO or water. All analyses were completed within $4 \mathrm{~h}$ after the blood collection. Drugs and chemical compounds were mainly purchased from Sigma.

\subsection{Hemorheological measurements}

Red blood cell aggregation in native plasma was assessed by the Myrenne aggregometer which provides an index of RBC aggregation facilitated by low shear. In brief, the suspension was subjected to a short period of high shear to disrupt pre-existing aggregates, following which the shear was abruptly reduced to $3 \mathrm{~s}^{-1}$ and light transmission through the suspension that was integrated for ten seconds; the resulting index, termed «M1» by the manufacturer and «RBCA» herein, increased with enhanced RBC aggregation.

Two methods were used to measure red blood cell deformability (RBCD):

1) Suspension viscosity of red blood cells (SV) was measured with capillary viscometer. The reproducibility of the capillary viscometer system was ranged between $1.0-1.5 \%$ as indexed by the coefficient of variation for 10 repeated measurements of the same sample.

2) A parallel plate flow channel was used to estimate red blood cell deformability (for details see: [1]). In brief, the cells were attached to bottom part of the chamber with "one point" and then they were deformed by shear flow, under constant shear stress. The length (L) and width (W) of each of about hundred cells were measured and elongation index (EI) as an index of red cell deformability was calculated according to: $\mathrm{EI}=(\mathrm{L}-\mathrm{W}) /(\mathrm{L}+\mathrm{W})$, using special computer program.

\subsection{Miscellaneous techniques}

Red blood cell suspension hematocrits were determined via the microhematocrit methods (i.e., $12,000 \times \mathrm{g}$ for 7 minutes). All analyses were completed within $4 \mathrm{~h}$ after the blood collection, at room temperature, about $200 \mathrm{C}$.

\subsection{Statistics and data presentation}

The results are presented as mean \pm standard deviation (SD). Comparisons were made using paired $t$-tests, and a two-tailed $p$ value of $p<0.05$ was considered to be significant; given the exploratory nature of these studies, $p$ values are reported as determined.

\section{Results}

Microrheological characteristics for patients prior to and after incubation with cisplatin and 5-FU presented in Table 1. Cisplatin increased markedly RBCD and both RBCA and red cell suspension viscosity were decreased. It was found $17 \%$ rise of red cell deformability and $24 \%$ drop in cell aggregation $(p<0.05)$. As for 5 -FU it was shown small positive RBCD effect together with a significant increase of RBCA by $12 \%(p<0.05)$.

The drug for the targeted antitumor therapy sunitinib raised red cell aggregation by $22 \%(p<0.05)$ together with the red cell suspension viscosity increase by $13 \%$ (Table 2). RBC deformability index (EI) was altered only slightly after cell incubation with sunitinib. Its decrease was less than $3 \%$ and was not statistically significant. 
Table 1

The RBC microrheological alterations after cell incubation with anticancer drugs: cisplatin and 5-fluorouracil (5-FU) $(\mathrm{M} \pm \mathrm{SD} ; n=30)$

\begin{tabular}{lccc}
\hline Parameter & Control & $\begin{array}{c}\text { Cisplatin } \\
(3.0 \mu \mathrm{M})\end{array}$ & 5-FU $(5.0 \mu \mathrm{M})$ \\
\hline Suspension viscosity, mPa·s & $3.42 \pm 0.21$ & $3.02 \pm 0.16^{*}$ & $3.28 \pm 0.16$ \\
RBCD & $0.242 \pm 0.019$ & $0.288 \pm 0.031^{*}$ & $0.244 \pm 0.028$ \\
RBCA & $8.72 \pm 0.36$ & $6.61 \pm 0.44^{*}$ & $9.79 \pm 0.56^{*}$ \\
\hline
\end{tabular}

5-FU - 5-fluorouracil; RBCA - red blood cell aggregation; RBCD - red blood cell deformability; ${ }^{*} p<0.05$ versus control.

Table 2

The RBC microrheological alterations after cell incubation with targeted drug sunitinib and epoetin alfa (M $\pm \mathrm{SD} ; n=30)$

\begin{tabular}{lccr}
\hline Parameter & Control & $\begin{array}{c}\text { Sunitinib } \\
(1.0 \mu \mathrm{M})\end{array}$ & $\begin{array}{c}\text { Epoetin alfa } \\
(10 \mathrm{M} . \mathrm{E} . / \mathrm{MJI})\end{array}$ \\
\hline Suspension viscosity, $\mathrm{mPa} \cdot \mathrm{s}$ & $3.45 \pm 0.18$ & $3.90 \pm 0.17^{*}$ & $2.92 \pm 0.16^{*}$ \\
RBCD & $0.252 \pm 0.023$ & $0.249 \pm 0.031$ & $0.282 \pm 0.030^{*}$ \\
RBCA & $8.14 \pm 0.44$ & $9.92 \pm 0.40^{*}$ & $7.33 \pm 0.32^{*}$ \\
\hline
\end{tabular}

RBCA - red blood cell aggregation; RBCD - red blood cell deformability, ${ }^{*} p<0.05$ versus control.

After RBC incubation with epoetin alfa it was found statistically significant increase in RBC deformability (Table 2$)$. Its rise was by $12 \%(p<0.05)$ together with RBC suspension viscosity and RBCA decrease by 15 and $10 \%(p<0.05)$ accordingly.

\section{Discussion}

At present it is known the mature erythrocytes retain a number of molecular components of signaling and/or regulatory pathways [12-14]. Of these, particular interest has been focused on erythrocyte protein kinases and phosphatases, because of their possible roles in the modulation of erythrocyte morphology, deformability and metabolic functions $[12,15,17,24]$. In vitro study showed that cisplatin has a direct positive microrheological effect. It can be supposed that cisplatin acts on RBC membrane molecular target. It is known that red cell membrane contains tyrosine protein kinase (TPK) $[3,4,18]$. Therefore cisplatin can activate these enzymes (Lyn protein tyrosine kinase of Src family [20, 23, 30, 32]). Indeed, there was a significant RBCA decrease together with RBCD rise after cisplatin treatment (Fig. 1A and 1B). In our observations it was detected that this specific TPK inhibitor lavendustin A eliminated positive changes of RBCA and RBCD (Fig. 1).

This compound induces protein tyrosine phosphorylation of numbers of cellular proteins, which suggests involvement of protein tyrosine kinases in the activation process of red cell membrane [20, $23,30,32]$. The protein kinase activation is expected to lead to a dissociation of ternary complex (band 3 - band $4.1 \mathrm{R}$ - spectrin) and an increase of red cell membrane plasticity (Fig. 2) [12, 26]. In addition it was also observed that $\mathrm{Ca} 2+/$ calmodulin and calmodulin-dependent kinases are involved in the regulation of cisplatin-induced Lyn expression and activation in erythrocytes [5, 24].

It is known that any changes in intracellular $\mathrm{Ca} 2+$ in erythrocytes are accompanied by red cell aggregation alterations [17]. This change mechanism was probably involved in the marked RBCA alteration after cell incubation with 5-FU. Our experimental data indicated that the chelation of $\mathrm{Ca} 2+$ 
A

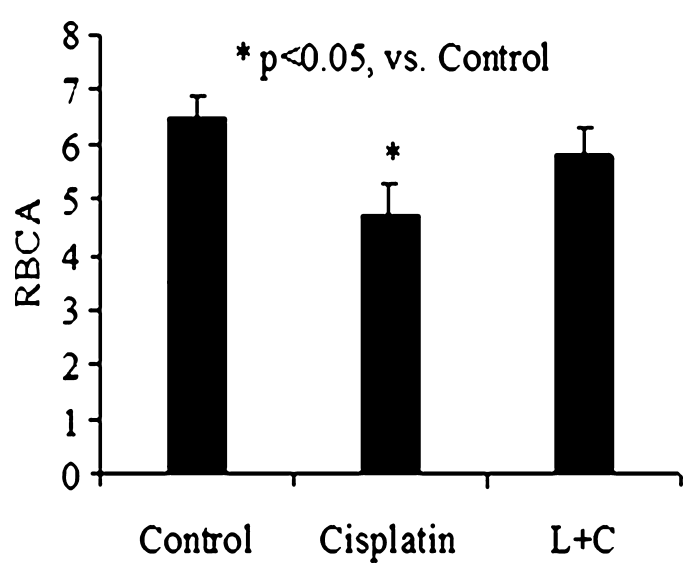

B

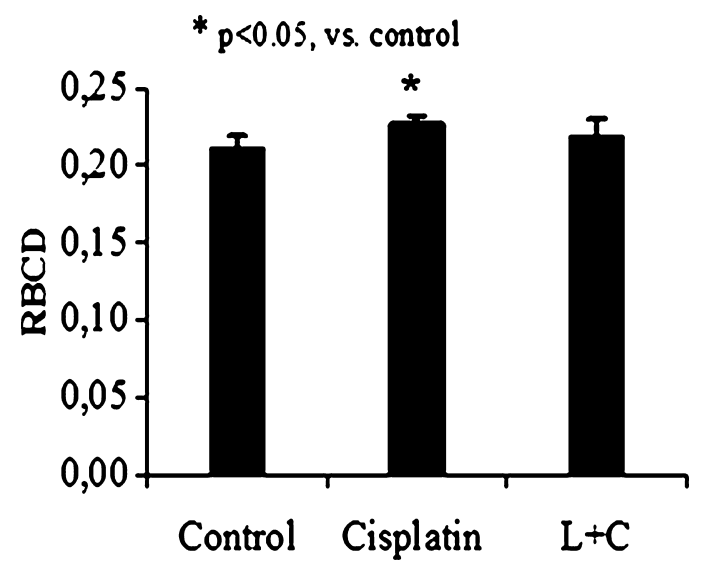

Fig. 1. The alteration of RBCA (A) and RBCD (B) under their incubation with cisplatin and with drug combination of lavendastin and cisplatin. (RBCA - red blood cell aggregation; RBCD - red blood cell deformability; L - lavendastin A; C - cisplatin).

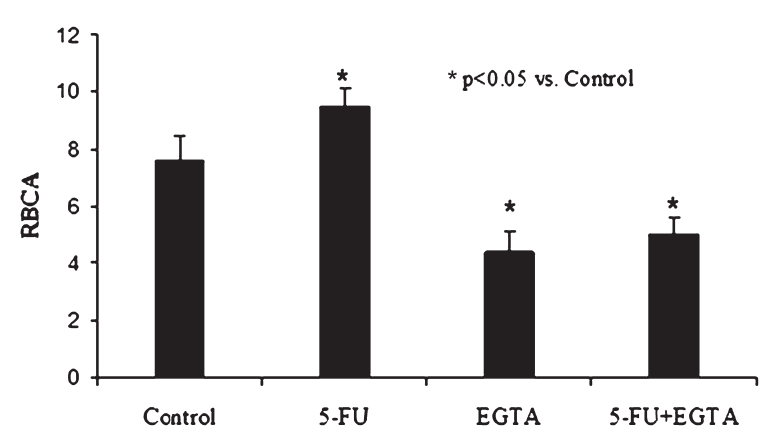

Fig. 2. The alteration of RBCA after cell incubation with 5-fluorouracil (5-FU) and with drug combination of 5-FU and EGTA $\left(10^{-3} \mathrm{M}\right)$. (RBCA - red blood cell aggregation).

in the cell incubation medium by EGTA caused a significant decrease RBCA followed by its significant rise due to 5-FU application (Fig. 2).

A lowered red cell aggregation effect was also observed when RBCs were incubated with pentoxifylline and what is more the latter almost removed proaggregation activity of 5-FU (Fig. 3).

Taken as a whole RBCA reduction was on the averaged $25 \%$ after cell incubation with pentoxifylline. An application of two drug combination (5-FU and pentoxifylline) was accompanied by $16 \%$ RBC aggregation lowering ( $p<0.05$, vs. Control, Fig. 4$)$.

The antitumor targeted drug - sunitinib did not significant change red cell deformability whereas RBCA was increased markedly after cell incubation with it (Fig. 4). As stated above cisplatin, as a stimulator of the TPK $[9,24]$. It was not so strange the combination of two these chemicals was accompanied by an elimination of proaggregative effect of sunitinib (Fig. 4). On the other hand the red cell deformability was decreased a little after sunitinib incubation, whereas after its combination with TPK stimulator cisplatin RBCD was a significant rise by $7 \%(p<0.05$, Fig. 5).

It is very likely that cisplatin is strong enough to activate TPK in erythrocyte membrane in spite of the tyrosine kinase inhibitor presence or may be sunitinib is mostly acts as an inhibitor of vascular endothelial growth factor in this case [28]. Hypertension is one of the most common adverse effects of angiogenesis inhibitors [10] and so the of RBCA rise after cell treatment with sunitinib may increase 


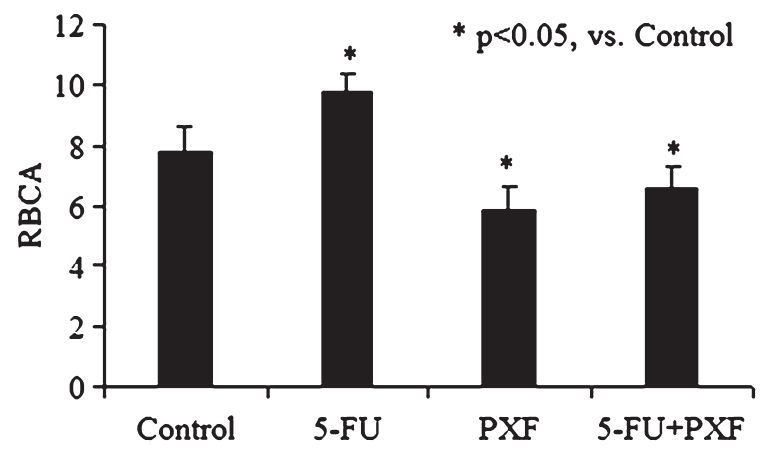

Fig. 3. The alteration of RBCA after cell incubation with 5-fluorouracil (5-FU), pentoxifylline (PXF) and with drug combination of 5-FU and pentoxifylline.

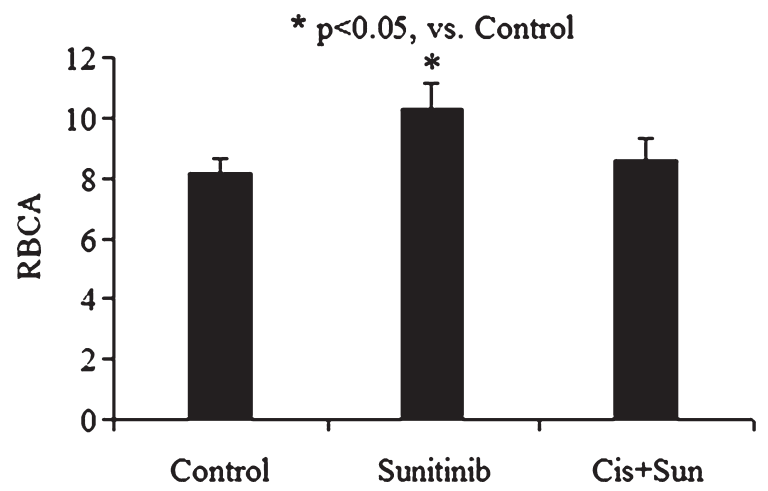

Fig. 4. The alteration of RBCA after cell incubation with sunitinib and with drug combination of sunitinib (Sun) and cisplatin (Cis).

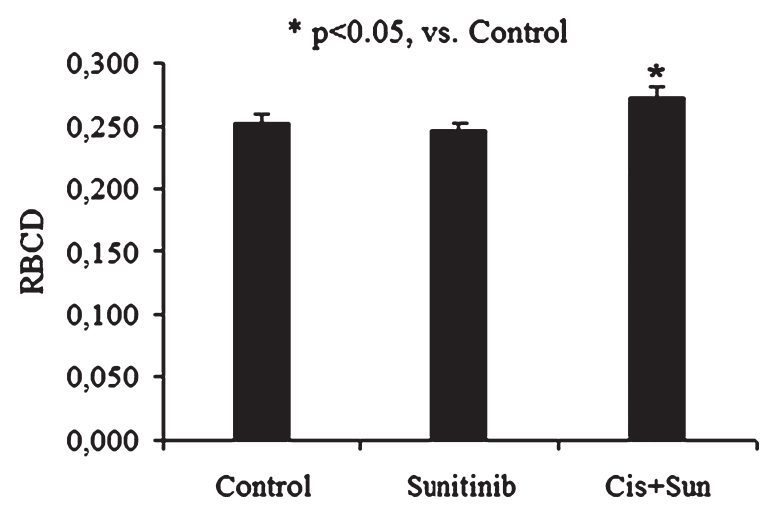

Fig. 5. The alteration of RBCD after cell incubation with sunitinib and with drug combination of sunitinib (Sun) and cisplatin (Cis).

attention to change in the RBC aggregability as a mechanism [11] involved in these process in the early stages of hypertension development.

The positive microrheological effect of epoetin alfa is probably connected with an activation many distinct signal transduction cascades, including a stimulation of tyrosine kinase - JAK-kinases [2, 5]. These chemicals induce protein tyrosine phosphorylation of a number of cellular proteins, which 
suggests involvement of TPK in the activation process of RBC membrane band 3 [27, 31]. The latter is expected to lead to a dissociation of complex (band 3 - ankyrin - spectrin) and to an increase of red cell membrane plasticity $[12,21]$.

Examination of the effects of some antitumor drugs on red blood cell microrheological properties has enabled us to suggest that the observed changes in red cell aggregation and deformability may be partly related to the effects of tyrosine protein kinase activation. It is accompanied by the alteration of red blood cell microrheology and the blood transport efficacy.

\section{Acknowledgments}

This work was supported by RSCF research project No. 14-15-00787.

\section{References}

[1] Artmann GM. Microscopic photometric quantification of stiffness and relaxation time of red blood cells in a flow chamber, Biorheology 1995;32:553-70.

[2] Baciu I, Pavel T, Marina C, Zirbo M, Zdrenghea C. Erythropoietin binding to the red cell membranes, Physiologie 1985;22:227-31.

[3] Brunati M, Bordin L, Clari G, Moret V. The Lyn-catalyzed Tyr phosphorylation of the transmembrane band 3 protein of human erythrocytes, Eur J Biochem 1996;240:394-99.

[4] Ciana A, Minetti G, Balduini C. Phosphotyrosine phosphatases acting on band 3 in human erythrocytes of different age: PTP1B processing during cell ageing. Bioelectrochemistry 2004;62:169-73.

[5] Chin H, Arai A, Wakao H, Kamiyama R, Miyasaka N, Miura O. Lyn physically associates with the erythropoietin receptor and may play a role in activation of the Stat5 pathway. Blood 1998;91:3734-45.

[6] Hartmann JT, Kanz L. Sunitinib and periodic hair depigmentation due to temporary c-KIT inhibition, Arch Dermatol 2008;144:1525-6.

[7] Hudis CA, Vogel CL, Gralow JR, Williams D. Procrit Study Group, Weekly epoetin alfa during adjuvant chemotherapy for breast cancer: Effect on hemoglobin levels and quality of life, Clin Breast Cancer 2005;6:132-42.

[8] Jung F, Mrowietz C, Hiebl B, Franke RP, Pindur G, Sternitzky R. Influence of rheological parameters on the velocity of erythrocyte passing nailfold capillaries in human, Clin Hemorheol Microcirc 2011;48:129-39.

[9] Kumar R, Shrivastava A, Sodhi A. Cisplatin stimulates protein tyrosine phosphorylation in macrophages, Biochem Mol Biol Int 1995;35:541-47.

[10] León-Mateos L, Mosquera J, Aparicio L. Antón. Treatment of sunitinib-induced hypertension in solid tumor by nitric oxide donors. Redox Biology 2015;6:421-25.

[11] Lominadze D, Joshua IG, Schuschke DA. Increased erythrocyte aggregation in spontaneously hypertensive rats, Am J Hypertens 1998;11:784-9.

[12] Manno S, Takakuwa Y, Mohandas N. Modulation of erythrocyte membrane mechanical function by protein 4.1 phosphorylation, J Biol Chem 2005;280:7581-7.

[13] Metere A, Iorio E, Pietraforte D, Podo F, Minetti M. Peroxynitrite signaling in human erythrocytes: Synergistic role of hemoglobin oxidation and band 3 tyrosine phosphorylation. Arch Biochem Biophys 2009;484:173-82.

[14] Minetti G, Piccinini G, Balduini C, Seppi C, Brovelli A. Tyrosine phosphorylation of band 3 protein in Ca2+/A23187treated human erythrocytes, Biochem J 1996;320:445-50.

[15] Mohandas N, Gallagher PG. Red cell membrane: Past, present, and future, Blood 2008;12:3939-48.

[16] Muravyov AV, Cheporov SV, Kislov NV, Volkova EL. Hemorheological changes in solid tumor patients after treatment with recombinant erythropoietin, Clin Hemorheol Microcirc 2009;41:39-47.

[17] Muravyov AV, Tikhomirova IA. Role Ca2+ in Mechanisms of the Red Blood Cell Microrheological Changes, In: Calcium Signaling, Md. Shahidul Islam ed, Springer Science+Buisness Media, Dordrecht; 2012;1017-37.

[18] Perlman DF, Musch MW, Goldstein L. Cell membrane surface expression and tyrosine kinase regulate the osmolyte channel (skAE1) in skate erythrocytes. Acta Physiol (Oxf) 2006;187:87-91.

[19] Pribush A, Hatskelzon L, Mazor D, Katorza E, Zilberman-Kravits D, Meyerstein N. The role of erythrocyte aggregation in the abnormal hemorheology of multiple myeloma patients, Clin Hemorheol Microcirc 2006;34:529-36. 
[20] Roskoski RJr, Src kinase regulation by phosphorylation and dephosphorylation, Biochem Biophys Res Commun 2005;331:1-14.

[21] Salomao M, Zhang X, Yang Y, Lee S, Hartwig JH, Chasis JA, Mohandas N, An X. Protein 4.1R-dependent multiprotein complex: New insights into the structural organization of the red blood cell membrane, Proc Natl Acad Sci USA 2008;105:8026-31.

[22] Siddik ZH. Cisplatin: Mode of cytotoxic action and molecular basis of resistance, Oncogene 2003;20(22):7265-79.

[23] Singh RA, Sodhi A. Expression and activation of lyn in macrophages treated in vitro with cisplatin: Regulation by kinases, phosphatases and Ca2+/calmodulin, Biochim Biophys Acta 1998;21:171-79.

[24] Singh RA, Sodhi A, Expression and activation of RAS and mitogen-activated protein kinases in macrophages treated in vitro with cisplatin: Regulation by kinases, phosphatases and Ca2+/calmodulin, Immunol Cell Biol 1999;77:356-63.

[25] Songdej N, von Mehren M, GIST treatment options after tyrosine kinase inhibitors, Curr Treat Options Oncol 2014;15:493-506.

[26] Takakuwa Y. Protein 4.1, a multifunctional protein of the erythrocyte membrane skeleton: Structure and functions in erythrocytes and nonerythroid cells, Int J Hematol 2000;72:298-309.

[27] Tong W, Zhang J, Lodish HF. Lnk inhibits erythropoiesis and Epo-dependent JAK2 activation and downstream signaling pathways, Blood 2005;154:604-12.

[28] Vázquez Sergio, León Luis, Ovidio Fernández, Lázaro Martín, Grande Enrique, Aparicio L, Sunitinib: The First to Arrive at First-Line Metastatic Renal Cell Carcinoma, Advances in Therapy 2012;29:202-17.

[29] Wang G, Reed E, Li QQ. Molecular basis of cellular response to cisplatin chemotherapy in non-small cell lung cancer (Review), Oncol Rep 2004;12:955-65.

[30] Yoshimura A, Arai K, Physician Education: The Erythropoietin Receptor and Signal Transduction, Oncologist 1996;1: 337-39.

[31] Yannoukakos D, Vasseur C, Piau JP, Wajcman H, Bursaux E. Phosphorylation sites in human erythrocyte band 3 protein, Biochim Biophys Acta 1991;1061:253-66.

[32] Zipser Y, Kosower NS. Phosphotyrosine phosphatase associated with band 3 protein in the human erythrocyte membrane, Biochem J 1996;15(314):881-7. 